\title{
Clinical, Dermatoscopic, and Histological Findings in a Diagnosis of Pityriasis Lichenoides
}

\author{
Dillon D. Clarey ${ }^{1}$, Scott R. Lauer ${ }^{2}$, Ryan M. Trowbridge ${ }^{1}$ \\ 1. Dermatology, University of Nebraska Medical Center, Omaha, USA 2. Pathology, University of Nebraska Medical \\ Center, Omaha, USA
}

Corresponding author: Ryan M. Trowbridge, ryan.trowbridge@unmc.edu

\begin{abstract}
Pityriasis lichenoides et varioliformis acuta (PLEVA) is a rare cutaneous eruption of erythematous macules and papules distributed over the flexural surfaces and the trunk. Histopathologic analysis is useful in diagnosis, and dermoscopic findings have been described in several small case series. We present a case of a mid-20s female who was diagnosed with PLEVA based on clinical and histopathological findings, and we also demonstrate a unique dermoscopic finding. Additionally, we review the current literature detailing dermoscopy findings with associated histopathology in PLEVA and pityriasis lichenoides chronica (PLC).
\end{abstract}

Categories: Dermatology, Pathology

Keywords: pityriasis lichenoides, dermoscopy, histopathology

\section{Introduction}

Pityriasis lichenoides is a dermatologic diagnosis consisting of three main forms: pityriasis lichenoides et varioliformis acuta (PLEVA), pityriasis lichenoides chronica (PLC), and febrile ulceronecrotic MuchaHabermann disease (FUMHD). Histologically, PLEVA is characterized by focal changes to the epidermis and dermis [1]. A vacuolar interface dermatitis with a perivascular lymphocytic infiltrate involving the superficial vascular plexus that extends into the deep reticular dermis in a wedge-shaped pattern can be seen. Erythrocyte and lymphocyte extravasation into the epidermis and epidermal necrosis may also been seen [1,2]. Dermoscopy has proven most beneficial in the diagnosis of pigmented lesions, although several reports have described characteristic dermoscopy findings in both PLEVA and PLC [3-7]. Here, we present a case of PLEVA diagnosed with unique dermatoscopic findings.

\section{Case Presentation}

An Asian female in her mid-20s with no significant past medical history presented with a one-month history of an erythematous and pruritic rash preceded by an upper respiratory infection; anti-streptolysin O (ASO), throat culture, and monospot tests were not performed at that time. The rash initially started on her forearms and then spread to her upper arms, back, and stomach. She reported that the rash was pruritic, waxed and waned, and worsened at night. She denied any new medications, foods, soaps, detergents, or cohabitating with other itchy individuals. She had previously been treated unsuccessfully by primary care with oral methylprednisolone, hydrocortisone $1 \%$ cream, and permethrin. 


\section{Cureus}

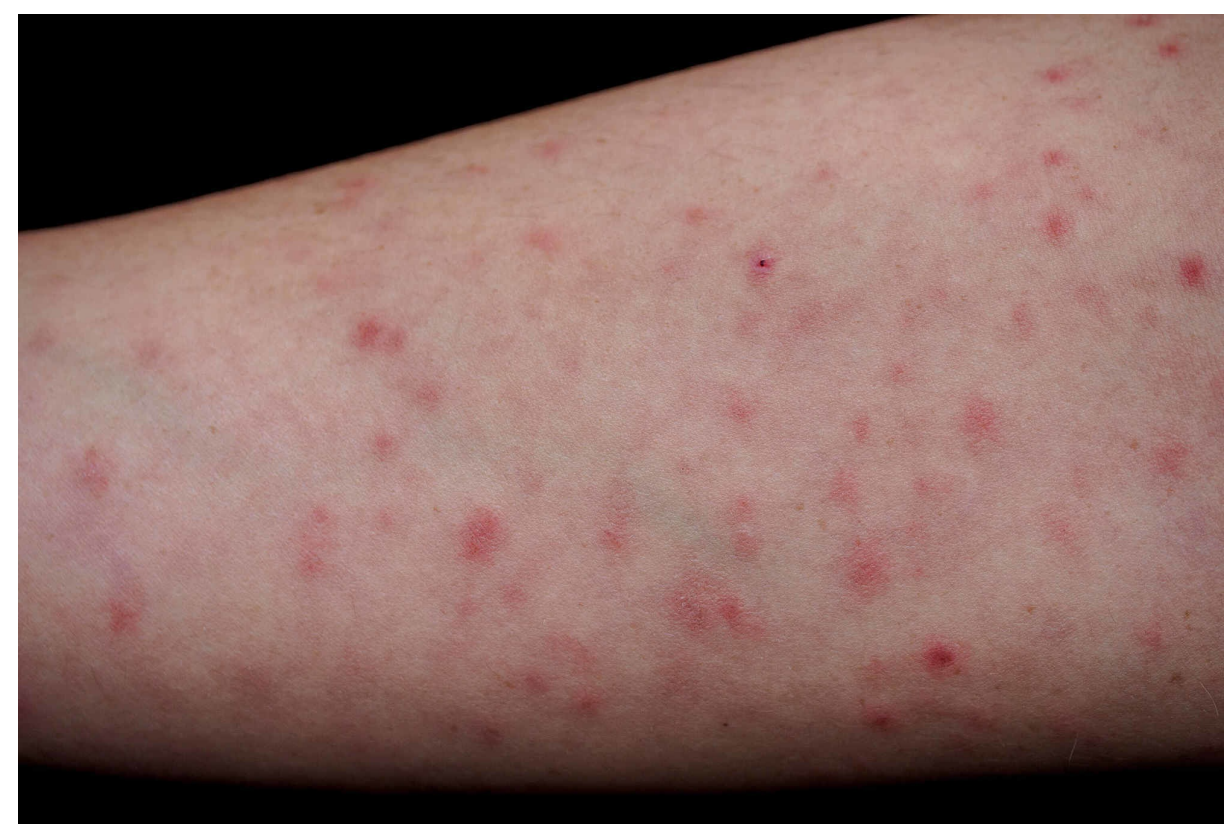

FIGURE 1: Red-purple-brown diffuse macules and papules with pigmented flecks distributed over the left volar forearm.

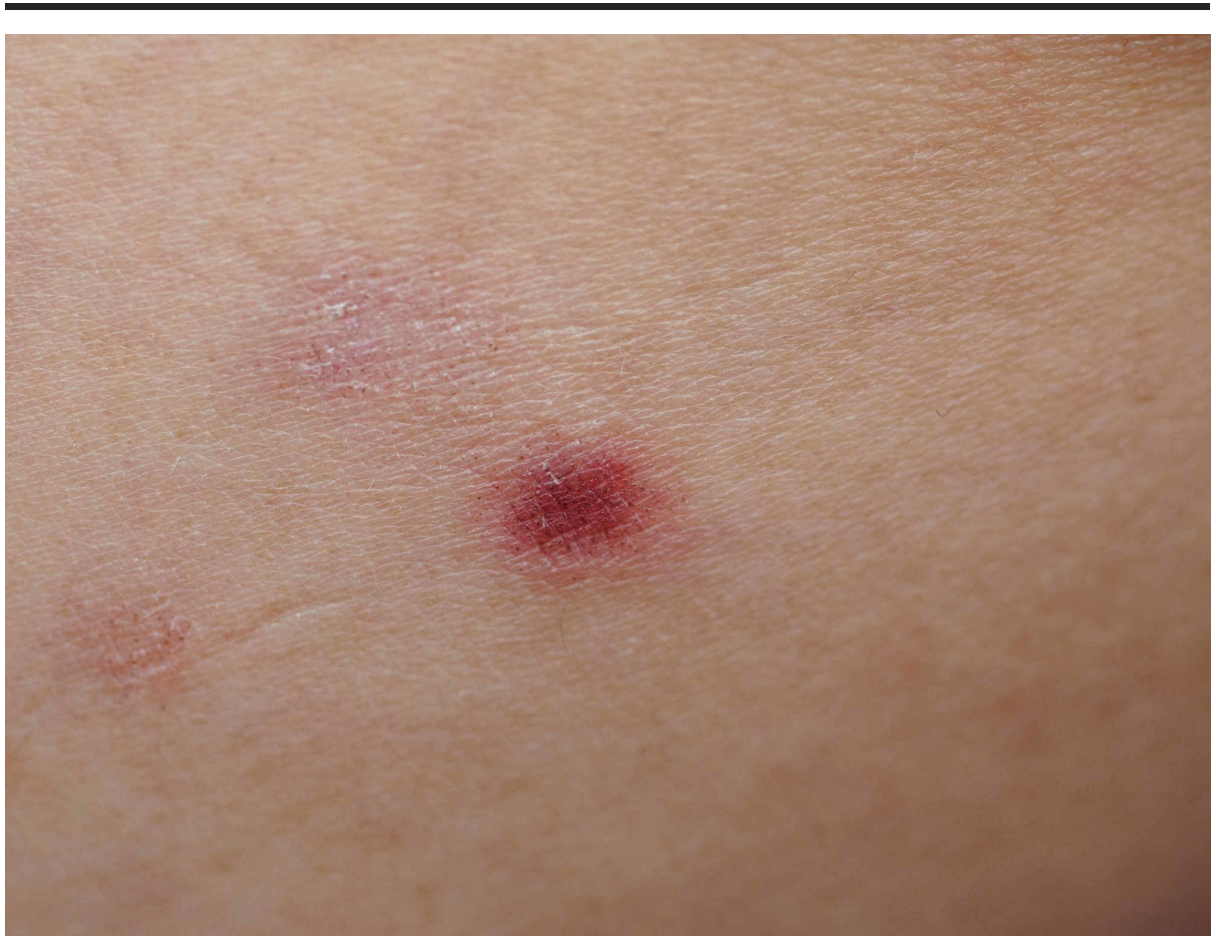

FIGURE 2: Close-up image of papule on the left volar forearm showing red thin papules with dark and light brown dots distributed within and outside of the red-purple coloration, respectively.

Under dermoscopy, a papule on the left volar forearm showed red thin papules with dark and light brown dots distributed within and outside of the red-purple coloration, respectively (Figure 3). 


\section{Cureus}

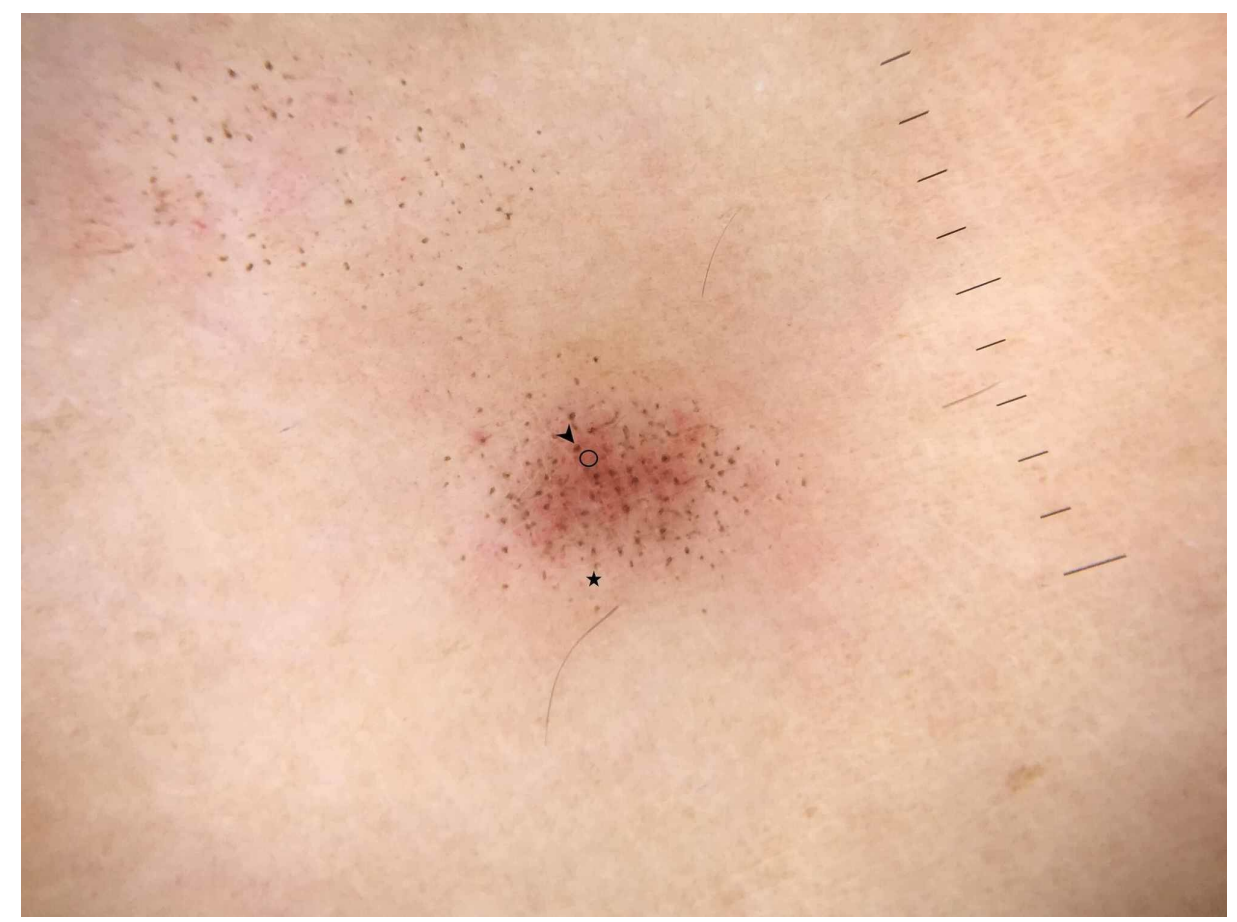

FIGURE 3: Dermoscopic view of papule in Figure 2 detailing pinpoint dark (black arrowhead) and light (black star) brown dots with underlying skin erythema (black circle) $(\times 10$, polarized; DermLite DL3N, 3Gen Inc, San Juan Capistrano, CA).

Punch biopsy from a papule on the right proximal calf was obtained and demonstrated vacuolar interface alteration with a mild superficial perivascular lymphocytic infiltrate and small numbers of extravasated erythrocytes (Figure 4). 


\section{Cureus}

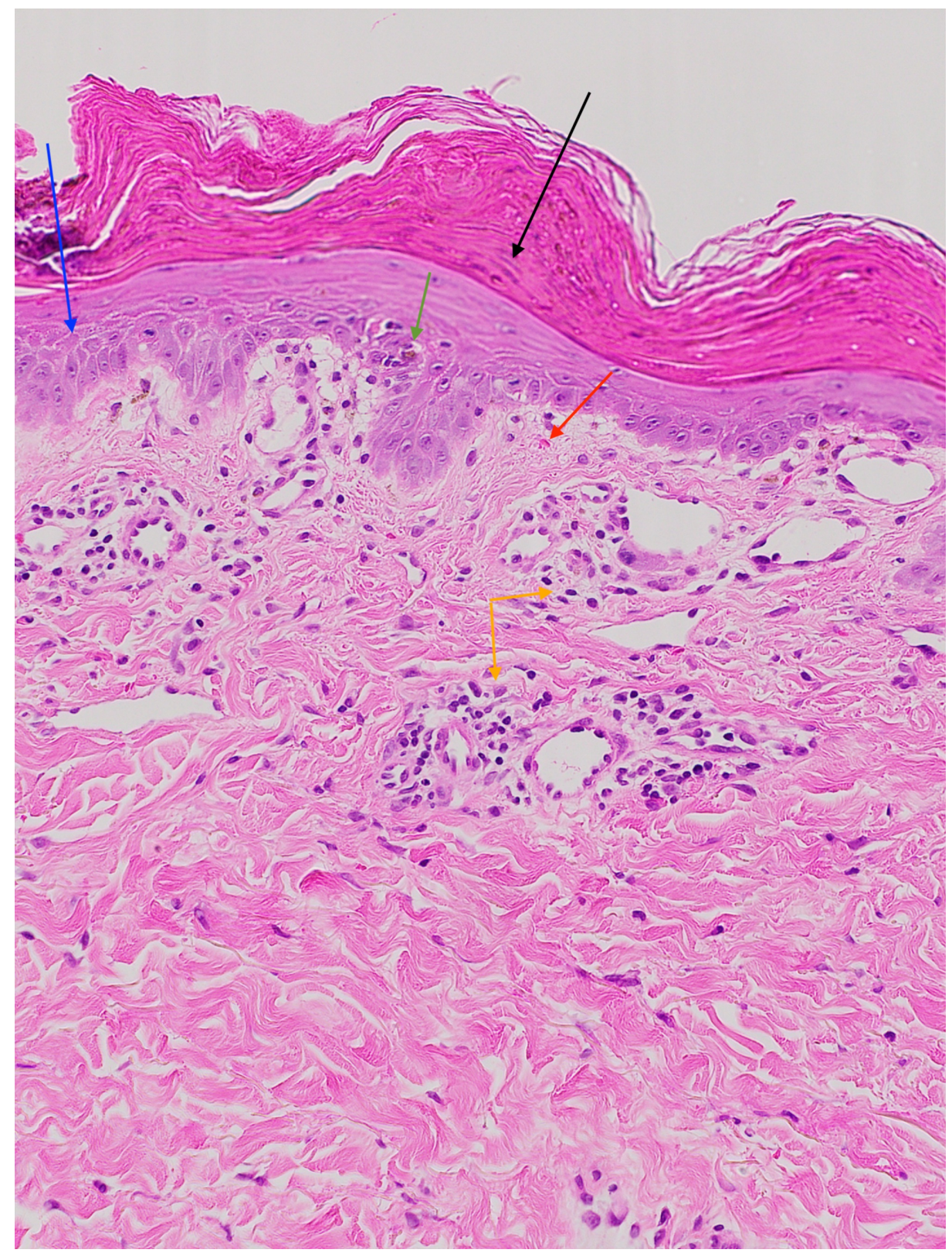

FIGURE 4: Focal parakeratosis (black arrow), mild spongiosis (blue arrow), mild superficial perivascular lymphocytic infiltrate (orange arrows), rare apoptotic keratinocytes (green arrow), and small numbers of extravasated erythrocytes (red arrow) (H\&E, ×20).

Periodic acid-Schiff (PAS) staining was performed and was negative for fungus. Fontana-Masson staining was performed and was significant for focal melanin deposition within the stratum corneum (Figure 5). Iron staining was performed and was negative. 


\section{Cureus}

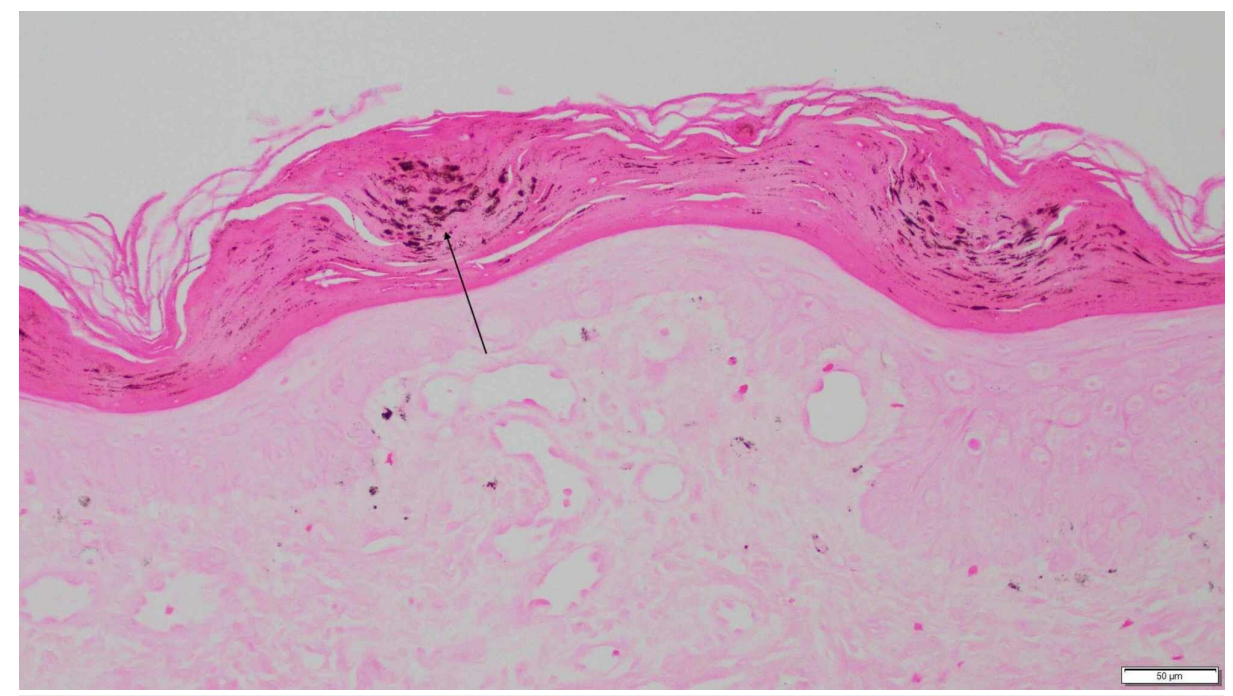

FIGURE 5: Focal melanin deposition within the stratum corneum (arrow) (Fontana-Masson, $\times 40$ ).

The findings were consistent with a clinical diagnosis of PLEVA. The patient was treated with doxycycline $100 \mathrm{mg}$ twice daily. The option of phototherapy in the future was discussed. At one-month follow-up, significant improvement was noted with a near-complete response on the trunk and mild, red, thin papules on the arms. She was to continue doxycycline $100 \mathrm{mg}$ twice daily with slow tapering off once no new lesions developed.

\section{Discussion}

Dermoscopy is most often utilized in the diagnosis of pigmented lesions. However, it has proven advantageous in the diagnosis of PLEVA due to its inexpensiveness, noninvasive technique, speed, and ability to differentiate from other diseases within the differential diagnosis [6]. A small number of reports have detailed PLEVA dermoscopy findings, with a 2010 report discussing individual cases of early and late phase lesions. The early phase lesion contained crusted brown and amorphous areas. The late phase lesion had a central white patch. In both cases, the lesions were surrounded by a ring of pinpoint/linear vascular structures with a targetoid aspect [3]. A 2016 study analyzed PLEVA in skin of color. Early phase lesions $(n=2)$ were again significant for unstructured brown areas surrounding hair follicles with central scale and dark red dotted vessels on the periphery [4]. These dotted areas were thought to be secondary to microhemorrhages, red blood cell (RBC) extravasation in the dermis, and blood vessel dilatation. Late lesions differed by containing a variety of appearances, notably blue-gray areas (dermal melanin), and yellow globules (basal cell degeneration, spongiosis) [4].

In our report, dermoscopy revealed red thin papules with light and dark brown dots distributed outside and within of the discoloration, respectively (Figure 3). Histopathology was significant for focal positive staining within the stratum corneum on Fontana-Masson in the location of these spots, indicative of focal melanin deposition (Figure 5). No previous reports of PLEVA have described melanin deposition in the stratum corneum. However, PLC has been noted to have flecks of melanin within the stratum corneum with associated parakeratosis [8].

With this in mind, our case seems to fit better histologically with PLC but clinically with PLEVA, highlighting the spectrum of presentation in pityriasis lichenoides. The explanation for this stratum corneum melanin deposition, notably the focality of it, remains elusive, as one would expect dermal hyperpigmentation secondary to disruption of the basal layer with the lichenoid infiltrate and subsequent descent of melanin and melanophages into the dermis [9]. These findings may be nonspecific and may be secondary to dyskeratosis.

Histologic and dermatoscopic findings (based on case reports) are key in the differentiation of PLEVA from other diagnoses within the clinical differential diagnosis (Table 1 ). 


\section{Cureus}

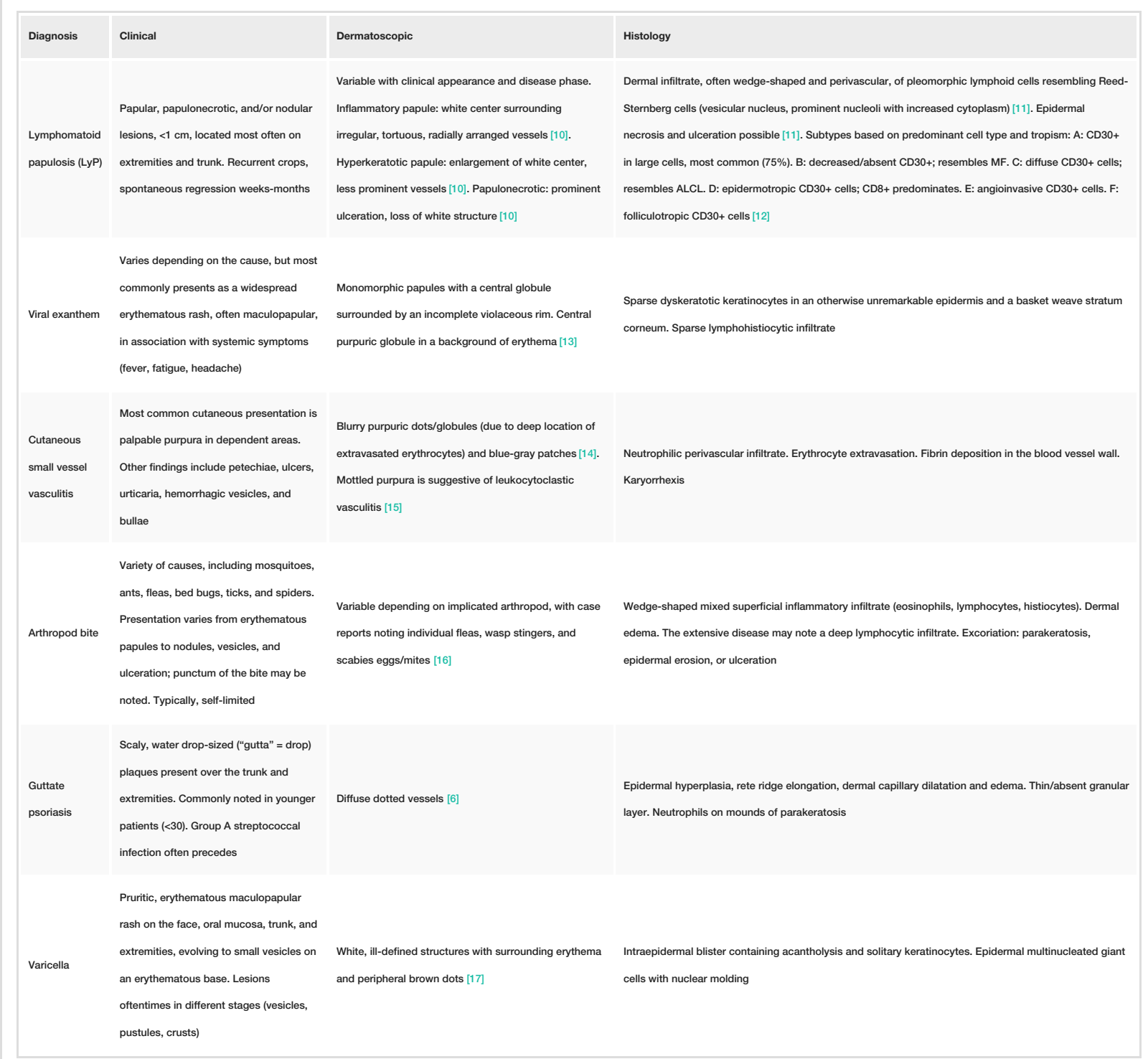

TABLE 1: Clinical differential diagnosis for PLEVA with associated dermatoscopic and histologic findings.

MF, mycosis fungoides; ALCL, anaplastic large cell lymphoma; PLEVA, pityriasis lichenoides et varioliformis acuta.

Without the utility of histologic and dermatoscopic findings illustrated in Table 1, several other important clues in the patient's presentation may assist in differentiating PLEVA from other diagnoses within its differential. For example, history including systemic symptoms (fever, fatigue, headache) may clue a provider towards a diagnosis of a viral exanthem. Organ-specific symptoms, including shortness of breath, cough, myalgias, and arthralgias, may raise clinical suspicion for vasculitis. Papulonecrotic or nodular lesions over the extremities and trunk raise clinical suspicion for lymphomatoid papulosis (LyP), notably important due to a roughly $15 \%$ risk of associated lymphoma (particularly mycosis fungoides [MF] and anaplastic large cell lymphoma [ALCL]) with LyP that is not present with PLEVA [18].

\section{Conclusions}

PLEVA is a cutaneous disorder that is at times challenging to diagnose clinically. Our case is unique to the literature in that it lacks the ring of pinpoint vasculature, brown amorphous areas, and white scale seen in other reports, suggesting that there may be a wide range of dermoscopic findings for PLEVA present within an individual case. We presented the unique finding of focal melanin deposition within the stratum corneum. Further, we detailed the clinical differential diagnosis of PLEVA and noted both dermatoscopic and histopathologic findings of diagnoses within this differential. Additional reports illustrating dermatoscopic findings and histopathology for pityriasis lichenoides may be beneficial in increasing diagnostic confidence for this challenging diagnosis. 


\section{Additional Information \\ Disclosures}

Human subjects: Consent was obtained by all participants in this study. Conflicts of interest: In compliance with the ICMJE uniform disclosure form, all authors declare the following: Payment/services info: All authors have declared that no financial support was received from any organization for the submitted work. Financial relationships: All authors have declared that they have no financial relationships at present or within the previous three years with any organizations that might have an interest in the submitted work. Other relationships: All authors have declared that there are no other relationships or activities that could appear to have influenced the submitted work.

\section{References}

1. Hood AF, Mark EJ: Histopathologic diagnosis of pityriasis lichenoides et varioliformis acuta and its clinical correlation. Arch Dermatol. 1982, 118:478-482. 10.1001/archderm.1982.01650190032014

2. Bowers S, Warshaw EM: Pityriasis lichenoides and its subtypes. J Am Acad Dermatol. 2006, 55:557-572. 10.1016/j.jaad.2005.07.058

3. Lacarrubba F, Micali G: Dermoscopy of pityriasis lichenoides et varioliformis acuta . Arch Dermatol. 2010, 146:1322. 10.1001/archdermatol.2010.263

4. Ankad BS, Beergouder SL: Pityriasis lichenoides et varioliformis acuta in skin of color: new observations by dermoscopy. Dermatol Pract Concept. 2017, 7:27-34.

5. Errichetti E, Stinco G: The practical usefulness of dermoscopy in general dermatology . G Ital Dermatol Venereol. 2015, 150:533-546.

6. Errichetti E, Lacarrubba F, Micali G, Piccirillo A, Stinco G: Differentiation of pityriasis lichenoides chronica from guttate psoriasis by dermoscopy. Clin Exp Dermatol. 2015, 40:804-806. 10.1111/ced.12580

7. Ankad B, Beergouder S: Dermoscopy of inflammatory conditions: the journey so far . EMJ Dermatol. 2017, 5:98-105.

8. Joshi R: Stratum corneum findings as clues to histological diagnosis of pityriasis lichenoides chronica . Indian J Dermatol Venereol Leprol. 2008, 74:156-157. 10.4103/0378-6323.39706

9. Attili S: "Interface" dermatoses: revisited. Indian J Dermatopathol Diagn Dermatol. 2019, 6:14-24. 10.4103/ijdpdd.ijdpdd_17_19

10. Moura FN, Thomas L, Balme B: Dermoscopy of lymphomatoid papulosis . Arch Dermatol. 2009, 145:966-966. 10.1001/archdermatol.2009.167

11. Martinez-Cabriales SA, Walsh S, Sade S, Shear NH: Lymphomatoid papulosis: an update and review. J Eur Acad Dermatol Venereol. 2020, 34:59-73. 10.1111/jdv.15931

12. Sundram U: Cutaneous lymphoproliferative disorders: what's new in the revised 4 th edition of the World Health Organization (WHO) classification of lymphoid neoplasms. Adv Anat Pathol. 2019, 26:93-113. 10.1097/PAP.0000000000000208

13. Larrondo J, Cabrera R, Gosch M, Larrondo F, Aylwin M, Castro A: Papular-purpuric exanthem in a COVID-19 patient: clinical and dermoscopic description [Epub ahead of print]. J Eur Acad Dermatol Venereol. 2020, 10.1111/jdv.16732

14. Choo JY, Bae JM, Lee JH, Lee JY, Park YM: Blue-gray blotch: a helpful dermoscopic finding in optimal biopsy site selection for true vasculitis. J Am Acad Dermatol. 2016, 75:836-838. 10.1016/j.jaad.2016.05.022

15. Ashfaq AM, Alon S, Vazquez-Lopez F, García-García B, Sanchez-Martin J, Argenziano G: Dermoscopic patterns of purpuric lesions. Arch Dermatol. 2010, 146:938. 10.1001/archdermatol.2010.162

16. Park JH, Kim CW, Kim SS: The diagnostic accuracy of dermoscopy for scabies . Ann Dermatol. 2012, 24:194199. 10.5021/ad.2012.24.2.194

17. Nayak S, Mehta H, Gajjar P, Nimbark V: Dermoscopy of general dermatological conditions in Indian population: a descriptive study. Clin Dermatol Rev. 2017, 1:41-51.

18. Melchers RC, Willemze R, Bekkenk MW, et al.: Frequency and prognosis of associated malignancies in 504 patients with lymphomatoid papulosis. J Eur Acad Dermatol Venereol. 2020, 34:260-266. 10.1111/jdv.16065 